\title{
Ibuprofen and the Phosphatidylcholine Bilayer: Membrane Water Permeability in the Presence and Absence of Cholesterol
}

Megan Wood, Michael Morales, Elizabeth Miller, Samuel Braziel, Joseph Giancaspro, Patrick

Scollan, Juan Rosario, Alyssa Gayapa, Michael Krmic, Sunghee Lee**

${ }^{1}$ Department of Chemistry, Iona College, 715 North Avenue, New Rochelle, New York 10801, USA

*To whom correspondence should be addressed. Tel: 914-633-2638. Fax: 914-633-2240.

E-mail: SLee@iona.edu

Supplementary Information

Figure S1 shows the Raman spectra of DOPC lipid bilayer (top) and ibuprofen (bottom) at room temperature. The detailed characteristic peak assignments for DOPC are shown in Table S1.

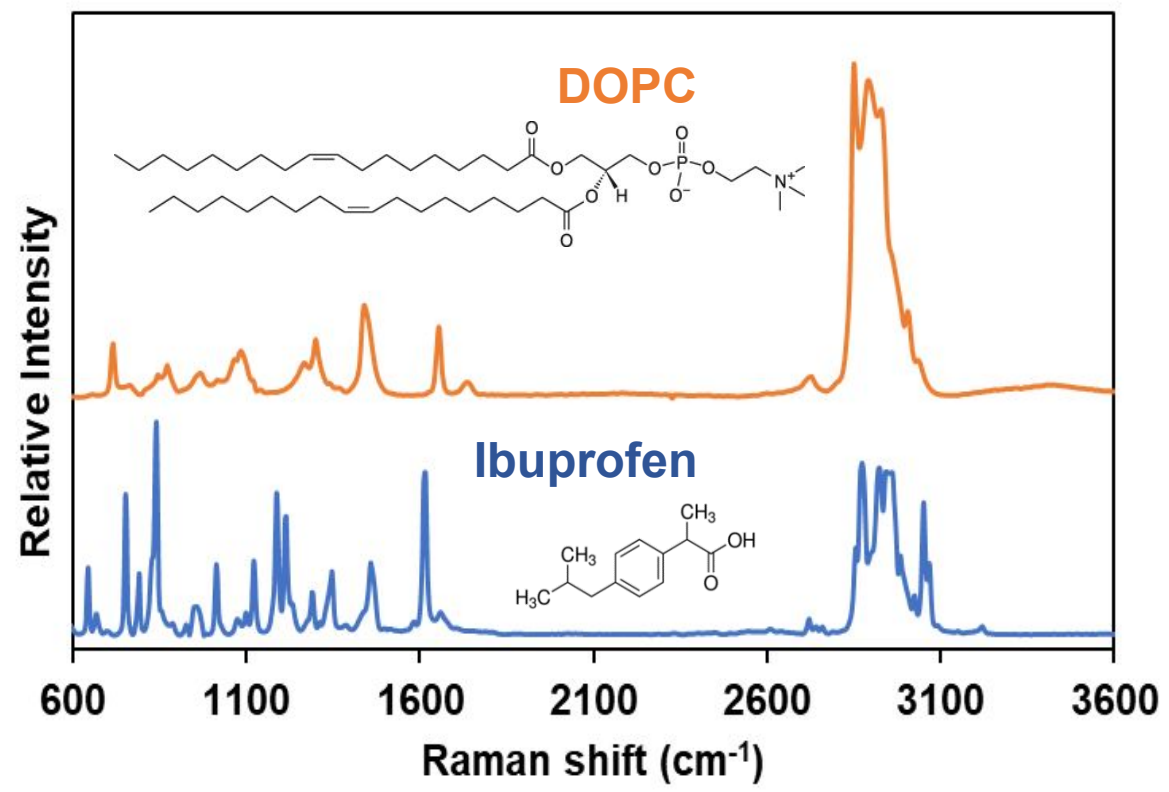

Figure S1. Raman spectra of pure DOPC lipid bilayer (film of pure DOPC liposome) and pure ibuprofen (dried film) at room temperature. 
Table S1. Peak assignments of DOPC Raman spectra ${ }^{1}$

\begin{tabular}{|c|c|}
\hline Raman peak $\left(\mathrm{cm}^{-1}\right)$ & Vibrational assignment \\
\hline 717 & $\mathrm{~N}-\mathrm{CH}_{3}$ symmetric stretch \\
\hline 872 & $\mathrm{~N}-\mathrm{CH}_{3}$ asymmetric stretch \\
\hline 1066 & $\mathrm{C}-\mathrm{C}$ trans stretch \\
\hline 1085 & $\mathrm{C}-\mathrm{C}$ gauche stretch \\
\hline 1299 & $\mathrm{CH}_{2}$ twist \\
\hline 1438 & $\mathrm{CH}_{2}$ bend \\
\hline 1655 & $\mathrm{C}=\mathrm{C}$ stretch \\
\hline 1735 & $\mathrm{C}=$ O stretch \\
\hline 2849 & $\mathrm{CH}_{2}$ symmetric stretch \\
\hline 2891 & $\mathrm{CH}_{2}$ asymmetric stretch \\
\hline 2928 & $\mathrm{CH}_{3}$ symmetric stretch \\
\hline 3004 & unsaturated C-H stretch \\
\hline 3034 & ${\mathrm{Choline} \mathrm{CH}_{3} \text { asymmetric stretch }}$ \\
\hline
\end{tabular}

Figure S2 shows Raman spectra of DOPC supported bilayers obtained from varying degree of addition of ibuprofen, at room temperature. All spectra are normalized to the intensity at 2849 $\mathrm{cm}^{-1}$ for comparison. A general trend of increasing intensity of peak at $1611 \mathrm{~cm}^{-1}$ (aromatic $\mathrm{C}-\mathrm{C}$ stretching from ibuprofen) is noticeable with increased concentration of ibuprofen in DOPC, along with other ibuprofen characteristic peaks (marked by red stars in Figure S2B). Figure S2B also shows the region of interests in the $\mathrm{C}-\mathrm{H}$ stretching $\left(2750-3050 \mathrm{~cm}^{-1}\right)$.

\footnotetext{
${ }^{1}$ Cherney, D.P.; Conboy, J.C.;Harris, J.M. Optical-trapping Raman microscopy detection of single unilamellar lipid vesicles. Analytical chemistry, 2003, 75(2 3), 6621-6628.
} 


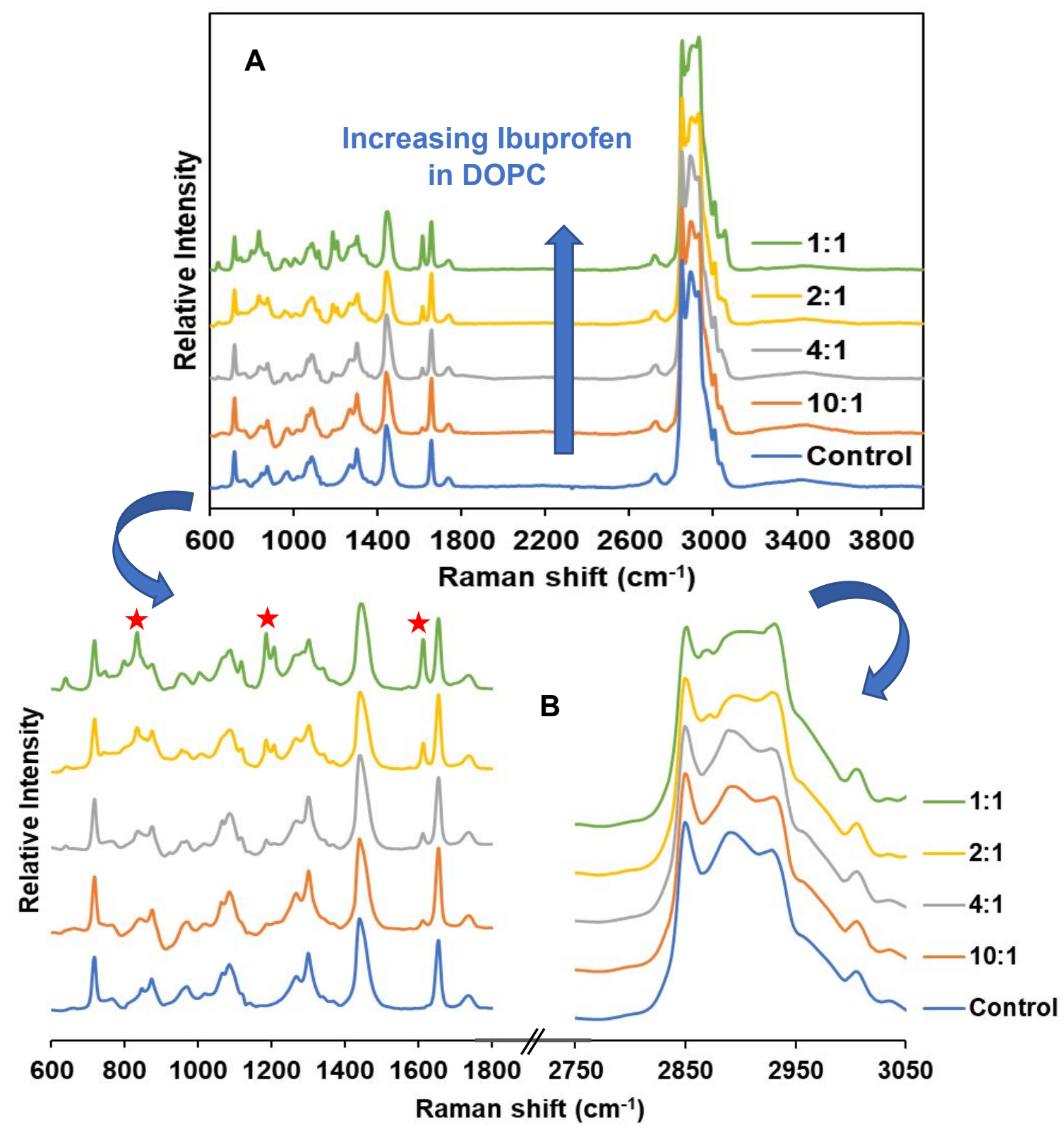

Figure S2. (A) Raman spectra of DOPC:ibuprofen (mol ratio) mixtures of increasing ibuprofen concentration at room temperature, (B) regions of interest in $600-1800 \mathrm{~cm}^{-1}$, and $2750-3050 \mathrm{~cm}^{-1}$, red stars indicate characteristic ibuprofen orginated peaks.

The $\mathrm{C}-\mathrm{H}$ stretching region $\left(2750-3050 \mathrm{~cm}^{-1}\right)$ also has peaks from ibuprofen that interfere with peaks from DOPC. Therefore, appropriate subtraction of ibuprofen peak is necessary to monitor 
the effect of ibuprofen on the packing properties of DOPC hydrocarbon chains. Before spectral subtraction, the ibuprofen spectra were scaled to the intensity of the $1611 \mathrm{~cm}^{-1}$ (aromatic $\mathrm{C}-\mathrm{C}$ stretching from ibuprofen). The resulting hydrocarbon chain contribution from DOPC is shown in black solid line in Figure S3A, along with before (dotted) subtraction of the scaled ibuprofen spectrum (solid red). The spectra shown in Figure S3B are those obtained after the spectral subtraction, allowing for the elimination of the ibuprofen components for varying mol fraction of ibuprofen in DOPC.

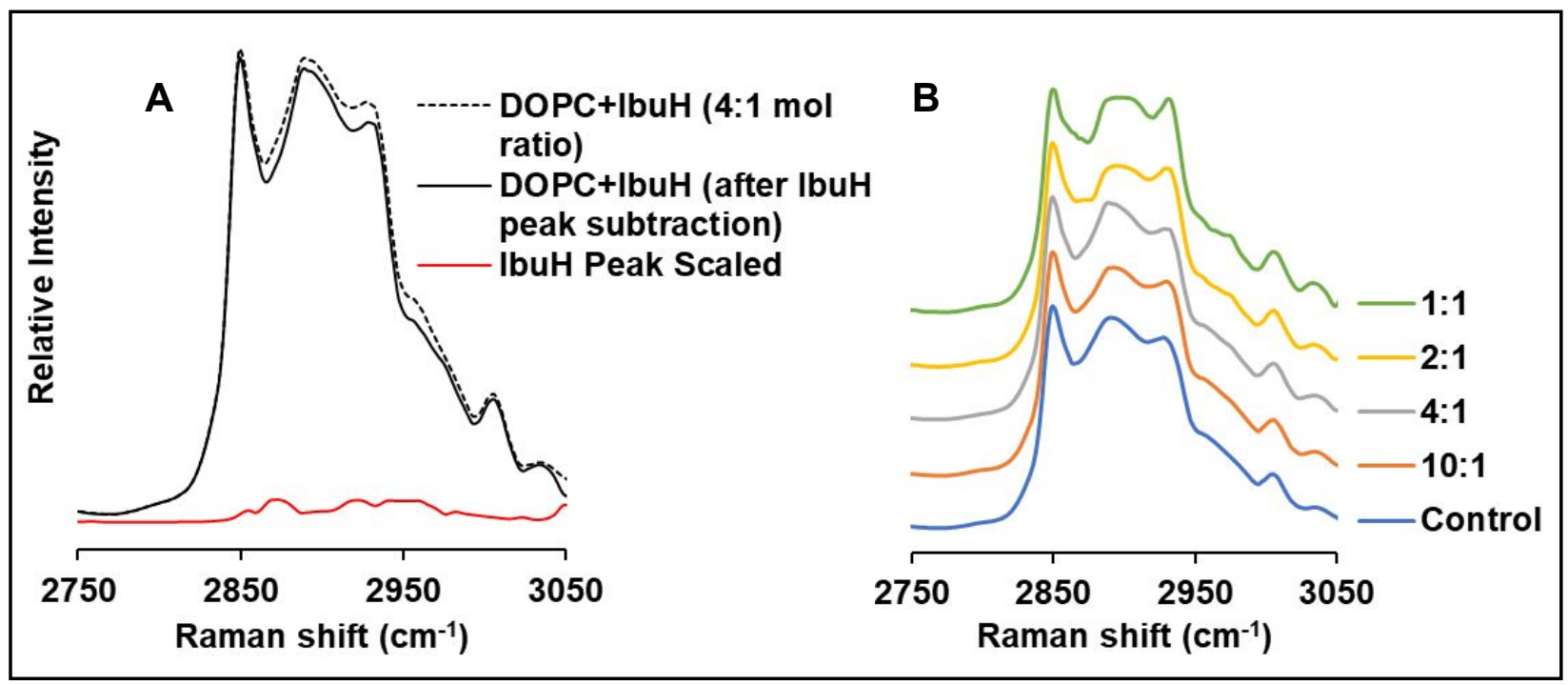

Figure S3. (A) Raman spectra of DOPC and ibuprofen mixture in the $\mathrm{C}-\mathrm{H}$ stretching region, the ibuprofen spectra are scaled to the intensity of the $1611 \mathrm{~cm}^{-1}$, the solid black line shows the spectrum of DOPC after subtraction of the ibuprofen spectrum (solid red line) from the original spectrum (dotted black line), (B) superposition of spectra of DOPC and ibuprofen lipid mixtures in the Raman shift region between 2750 and $3050 \mathrm{~cm}^{-1}$ after subtraction. 